\title{
Maternal Care Modulates the Relationship between Prenatal Risk and Hippocampal Volume in Women But Not in Men
}

\author{
Claudia Buss, ${ }^{1,2}$ Catherine Lord, ${ }^{2}$ Mehereen Wadiwalla, ${ }^{2}$ Dirk H. Hellhammer, ${ }^{1}$ Sonia J. Lupien, ${ }^{2}$ Michael J. Meaney, ${ }^{2}$ \\ and Jens C. Pruessner ${ }^{2}$ \\ ${ }^{1}$ University of Trier, Department for Theoretical and Clinical Psychobiology, 54290 Trier, Germany, and ${ }^{2}$ Douglas Hospital Research Center, Montreal, \\ Quebec, Canada H4H 1R3
}

\begin{abstract}
Smaller hippocampal volume is associated with psychiatric disorders. Variations in hippocampal volume are discussed as both a consequence of the neurotoxic effects of stress and as a pre-existing condition leading to increased vulnerability for cognitive and emotional impairments. To investigate whether early experience can account for variability in hippocampal volume in adulthood (vulnerability hypothesis), we assessed the relationship between birth weight and hippocampal volume in 44 subjects. The reported quality of maternal care in early childhood, as evaluated by the Parental Bonding Inventory, was used as index of the quality of the postnatal environment. Hippocampal volume was assessed from magnetic resonance images using a manual segmentation protocol. We show that birth weight significantly predicts hippocampal volume in adulthood only in female subjects reporting low maternal care. The results suggest that the postnatal environment modulates the neurodevelopmental consequences of prenatal risk and that this effect is sex-specific.
\end{abstract}

Key words: hippocampus; birth weight; maternal care; development; prenatal; postnatal

\section{Introduction}

Smaller hippocampal volumes have been reported in different clinical populations of patients suffering from psychiatric disorders (Geuze et al., 2005). Those variations in hippocampal volume, often accompanied by changes in endocrinological regulation, especially of the hypothalamic-pituitary-adrenal (HPA) axis, and impaired memory performance are considered either as a consequence of the neurotoxic effects of stress (Sapolsky et al., 1986) or as a pre-existing condition leading to increased vulnerability for cognitive and emotional impairments (Gilbertson et al., 2002). Several studies reveal the significance of prenatal and postnatal experience on hippocampal development, HPA functioning, and cognitive performance (Seckl, 2004). These studies suggest that the quality of parent-offspring interactions over the perinatal period can presumably affect hippocampal development.

Rodent studies provide evidence for postnatal compensation of prenatal adversity. Postnatal handling or increased maternal care can reverse the effects of prenatal stress or glucocorticoid administration on spatial memory performance, HPA stress response, and hippocampal GR expression (Brabham et al., 2000). Intriguingly, postnatal handling has been shown previously to

Received July 28, 2006; revised Jan. 23, 2007; accepted Jan. 24, 2007.

This work was supported in part by Canadian Institutes of Health Research (CIHR) Grants 67071 (J.C.P.) and 134254 (S.J.L.), and by Natural Sciences and Engineering Research Council of Canada Grant 249996 (J.C.P.). J.C.P. holds a New Investigator Award from CIHR. S.J.L. holds an Institute of Aging Investigator Award from CIHR. M.J.M. holds a Senior Investigator Award from CIHR and a Distinguished Investigator Award from the National Alliance for Research in Schizophrenia and Affective Disorders.

Correspondence should be addressed to Claudia Buss, University of Trier, Department for Clinical and Theoretical Psychobiology, Johanniterufer 15, 54290 Trier, Germany. E-mail: buss@uni-trier.de.

DOI:10.1523/JNEUROSCI.3252-06.2007

Copyright $\odot 2007$ Society for Neuroscience $\quad$ 0270-6474/07/272592-04\$15.00/0 prevent the prenatal stress-induced deficits in hippocampal neurogenesis (Lemaire et al., 2006).

Low birth weight in humans, reflecting intrauterine growth restriction potentially as result of a disadvantageous prenatal environment (Wadhwa et al., 1993), has been shown to be associated with alterations in HPA activity and increased rates of the metabolic syndrome (Phillips, 2004). However, follow-up studies of low-birth weight infants reveal considerable variability in outcome measures (Barker et al., 2002), suggesting that postnatal factors might interact with prenatal conditions. We hypothesized that in humans the development of the hippocampus might be a region of the brain that is influenced by both prenatal risk factors and the quality of the postnatal family environment.

\section{Materials and Methods}

Subjects were recruited by online advertisement on websites of local Universities. A total of 445 subjects were screened using telephone interviews and questionnaires. This prescreening consisted of information about their medical history and health status, including medication intake. Furthermore, it entailed information about birth outcomes (weight, length, and gestational age at birth), which had to be received from birth documents. Also, the Parental Bonding Inventory (PBI) (Parker et al., 1979) had to be completed, a questionnaire that retrospectively measures self-reported quality of parental bonding during the first 16 years of life. Subjects who suffered from chronic illness of any kind, including psychiatric disorders and those taking any medication (except oral contraceptives) were excluded from the study. Persons who had metal fragments, respectively implants or a pacemaker, as well as persons who reported to suffer from claustrophobia were advised against participation in the study because magnet resonance imaging (MRI) was involved in the study design.

Subjects were included in the study if they were born at term (gestational length $>37$ weeks, which was determined on the basis of the calculated due date). They were considered carrying a prenatal risk if they 
Table 1. Descriptives of subjects reporting high and low maternal care

\begin{tabular}{|c|c|c|c|}
\hline & High maternal care group $(n=22)$ & Low maternal care group $(n=22)$ & Statistical significance \\
\hline Mother care & $32.2( \pm 3.2)$ & $18.9( \pm 5.5)$ & $t_{(42)}=9.9 ; p=0.00$ \\
\hline Mother overprotection & $9.7( \pm 5.8)$ & $18.9( \pm 6.6)$ & $t_{(42)}=-4.9 ; p=0.00$ \\
\hline Father care & $26.8( \pm 7.1)$ & $20.1( \pm 7.1)$ & $t_{(41)}=2.8 ; p=0.00$ \\
\hline Father overprotection & $7.2( \pm 4.1)$ & $11.7( \pm 4.2)$ & $t_{(41)}=-3.5 ; p=0.00$ \\
\hline Birth weight (g) & $3140.6( \pm 431.78)$ & $3190.4( \pm 457.1)$ & $t_{(42)}=-0.37 ; p=0.7$ \\
\hline Birth length $(\mathrm{cm})$ & $50.5( \pm 4.1)$ & $51.2( \pm 3.5)$ & $t_{(41)}=-0.58 ; p=0.6$ \\
\hline Gestational length & $39.9( \pm 1.1)$ & $39.8( \pm 1.3)$ & $t_{(42)}=0.25 ; p=0.8$ \\
\hline
\end{tabular}

were born small for gestational age (SGA; birth weight was within the 10th percentile of the distribution) (Kramer et al., 2001) because high levels of maternal stress during pregnancy have been shown to impair fetal growth (Wadhwa et al., 1993). The selected reference group was born appropriate for gestational age (AGA; birth weight between the 40th and 70th percentile). Subjects born prematurely were excluded because it is known that prematurity has an impact on neurodevelopment (Abernethy et al., 2002) and we were interested in the impact of intrauterine growth restriction without this confounding factor.

Self-reported parental bonding, as assessed by the PBI (Parker et al., 1979), was applied as a proxy for interindividual variation in the quality of the postnatal environment. Therefore, postnatal risk in this study was characterized by the subjects' scores on the PBI, a questionnaire that retrospectively measures the self-reported quality of parental bonding during the first 16 years of life. The questionnaire consists of four scales (maternal care and overprotection and paternal care and overprotection). For each parent, 12 questions are asked in reference to "care" (e.g., "Spoke to me with a warm and friendly voice") and 12 questions in reference to "overprotection" (e.g., "Invaded my privacy"). Questions rating maternal and paternal parenting style are the same and are answered individually for each parent on a four-point Likert scale (very true to very untrue, $0-3$ ). For each scale a maximum score of 36 can be reached. The subjects were assigned to the postnatal risk groups based on their maternal care scores, using the suggested cutoff score of 27 by Parker and Lipscombe (1979). As shown in Table 1, the subjects of the postnatal risk group did not only report lower maternal care, but on average also reported lower paternal care as well as higher levels of maternal and paternal overprotection. As opposed to optimal parenting that is characterized by high levels of care and low levels of overprotection, Parker (1983) refers to this combination of low care and high overprotection as "affectionless control," which has been found to be highly predictive of depressive symptomatology. Although the two groups differed on each scale of the PBI, it is important to note that they did not differ in their birth outcomes (Table 1).

Because of application of the predefined prenatal and postnatal risk factors, the final selection included 44 subjects, constituting four groups (mean age, $23.32 \pm 3.2$ years). Twenty-one subjects were born small for gestational age and assigned to the prenatal risk group and 23 subjects were born AGA, constituting the reference group. Within the SGA group, 11 subjects reported high maternal care (six men and five women) and 10 subjects reported low maternal care (five men and five women). Among the subjects born AGA, 11 reported high maternal care (five men and six women) and 12 subjects reported low maternal care (five men and seven women).

There was a significant difference in age between the low and high maternal care groups ( $24.4 \pm 3.7$ vs $22.2 \pm 2.0$ years). Therefore, age was controlled for in all analyses performed to exclude a systematic effect of age.

As part of a functional MRI study investigating neurocorrelates of stress to be reported elsewhere, MRI scans were obtained using a Siemens (Erlangen, Germany) Magnetom 1.5 T system with a standard radiofrequency head coil. The volumes were acquired using a spoiled gradient echo sequence with a repetition time of $22 \mathrm{~ms}$, echo time of $10 \mathrm{~ms}$, flip angle of 30 , and field of view of $224 \times 256 \times 160 \mathrm{~mm}$. This protocol acquires T1-weighted images on $1 \mathrm{~mm}$ isotropic resolution. After acquisition, the native MR images were processed by a combination of algorithms for manual segmentation. The images were first corrected for intensity nonuniformity and then registered into Talairach-like space. This latter step accounts for individual differences in global brain size and shape. Images were classified into different maps of gray matter, white matter, and cerebrospinal fluid. This procedure includes the removal of all extracranial tissue and the cerebellum and has been validated before (Collins et al., 1994). Manual segmentation of the hippocampus was performed using a protocol developed by Pruessner et al. (2000). This protocol with proven reliability and validity has been implemented in numerous studies of this laboratory (Pruessner et al., 2001, 2005) and benefits from superior visualization of the target structures in all three dimensions (coronal, sagittal, and horizontal).

\section{Results}

A multivariate analysis of covariance (MANCOVA), controlling for age, was performed with left and right hippocampal volume as the dependent variables and prenatal and postnatal risk group as the independent variables. Subjects born small for gestational age did not differ in left or right hippocampal volume from those born appropriate for gestational age (left hippocampus, $F_{(1,40)}=$ $1.4, p=0.24$; right hippocampus, $\left.F_{(1,40)}=0.8, p=0.38\right)$. Neither did subjects who reported high maternal care differ in left or right hippocampal volume from those reporting low maternal care (left hippocampus, $F_{(1,40)}=0.33, p=0.57$; right hippocampus, $\left.F_{(1,40)}=0.12, p=0.7\right)$. A significant interaction between prenatal and postnatal risk groups was observed for right but not left hippocampal volume (left hippocampus, $F_{(1,40)}=2.57, p=0.12$; right hippocampus, $\left.F_{(1,40)}=4.19, p=0.05\right)$. The significant interaction was driven by significantly smaller hippocampal volumes in SGA subjects reporting low maternal care as compared with AGA subjects reporting low maternal care. In the high maternal care group, no differences in hippocampal volume could be observed in subjects born small as compared with those born appropriate for gestational age. Partial correlational analyses confirm these results. When controlling for length of gestation and age, a positive association between birth weight and left ( $r=$ $0.59 ; p=0.006)$, right $(r=0.51 ; p=0.02)$, and mean hippocampal volume $(r=0.57 ; p=0.009)$ was found in the group reporting low maternal care. In contrast, no such relation was found in the group reporting high maternal care (left hippocampus, $r=$ $0.15, p=0.54$; right hippocampus, $r=0.03, p=0.91$; mean hippocampus, $r=0.09, p=0.7)$. Because of the small sample size, the MANCOVA could not be repeated with sex as an additional factor. Therefore, correlational analyses were performed. Analysis of sex demonstrated that in the group of subjects reporting low maternal care, the positive relationship between birth weight and mean hippocampal volume was highly significant in the females $(r=0.76 ; p=0.01)$, whereas this correlation was not significant in the males $(r=0.26 ; p=0.54)$ (Fig. 1). Among subjects reporting high maternal care, there were no associations between birth weight and hippocampal volume in either females $(r=-0.36 ; p=0.35)$ or males $(r=0.15 ; p=0.69)$.

To investigate whether this interactive impact of prenatal and postnatal risk factors is specific to the hippocampus, the same 
partial correlations (controlling for length of gestation and age) were performed for total gray matter volume. These revealed that weight at birth did not correlate with total gray matter volume in the two groups differing in self-reported maternal care (high maternal care, $r=0.08, p=0.75$; low maternal care, $r=0.02, p=0.93$ ). Furthermore, performing separate analyses for males and females did not suggest an association between birth weight and total gray matter volume in either males (high maternal care, $r=0.15, p=0.7$; low maternal care, $r=0.37, p=0.36$ ) or females (high maternal care, $r=-0.21, p=$ 0.6 ; low maternal care, $r=-0.43, p=$ $0.25)$.

\section{Discussion}

In this study, we show that weight at birth predicts hippocampal volume but not total gray matter volume in a group of subjects reporting low maternal care. Thus, the results suggest the hippocampus as a distinct target for early parent-offspring interactions, which could be attributable to its protracted development over both prenatal and postnatal periods, high density of glucocorticoid receptors, and postnatal neurogenesis (Teicher et al., 2003). To our knowledge, this is the first study showing an association between birth weight and hippocampal volume in a healthy population without confounding perinatal medical complications. The fact that this association is only evident in the group reporting low maternal care suggests that the risks associated with fetal life are modulated by the quality of the postnatal environment. It is important to note that the two postnatal risk groups did not only differ on the maternal care scale of the PBI, but on the other three scales of the PBI as well. Therefore the group, referred to as having received low maternal care, is actually characterized by low parental bonding, respectively by parental "affectionless control" (Parker et al., 1979), so that the observed modulatory effect of the postnatal environment is most likely an effect of parental bonding in general instead of specifically maternal care.

An enhanced sensitivity of hippocampal development to the quality of the perinatal environment was observed in females. Such sex-specific effects have been reported in nonhuman subjects (Weinstock, 2005). In terms of hippocampal integrity, maternal stress during midgestation as well as during late gestation has been found to cause a significant decrease in the number of hippocampal neurons in the female, but not in the male offspring (Zhu et al., 2004). Such higher susceptibility to prenatal insult in female offspring has been discussed in the context of findings in mice, where female fetuses are exposed to higher baseline serum corticosterone concentrations than male fetuses because of greater transport of corticosterone from maternal blood across the placenta (Montano et al., 1993).

Additional support for sex-specific programming comes from human studies, where lower $11 \beta$-hydroxysteroid dehydrogenase type 2 (11 $\beta$-HSD2) concentrations associated with reduced fetal growth and higher cortisol concentration in the umbilical vein were observed in female but not in male offspring of mothers with untreated asthma (Murphy et al., 2003). Interestingly, maternal physiology seems to be affected by the fetus in a sex-specific way, because only in those asthmatic mothers carrying a female fetus, was an upregulation of inflammatory pathways observed (Murphy et al., 2003), whereas the same was not found in asthmatic mothers pregnant with a male fetus. Therefore, Murphy et al. (2003) discuss that the observed reduction of $11 \beta$-HSD2 activity in female offspring may be a result of increased maternal inflammation, suggesting that an unknown factor from the female fetus alters maternal immune function and thereby potentially affects its own growth. Although what this factor may be is not known, the authors suggest that it could be a sex steroid hormone or a novel protein originating from the fetus. The increased hippocampal sensitivity to the effects of early life experience in women could potentially be linked to depression, which has a higher prevalence in women and is associated with smaller hippocampal volumes (Vythilingam et al., 2002).

Although the reported effects of prenatal and postnatal risk factors on hippocampal volume were strong, there are a number of limitations in the current study. First, the study used a retrospective design. Causes of intrauterine growth restriction are likely diverse, and although the description of birth phenotype can be considered accurate, there are likely other factors which we were unable to capture with the current approach. However, size at birth has been used as an indicator of an adverse prenatal environment in a number of studies, and a relationship with disease susceptibility in later life has been repeatedly shown (Phillips, 1998; Reynolds et al., 2001; Kajantie et al., 2002). Second, measuring the quality of the postnatal environment with a retrospective questionnaire is limiting as well. Certainly, the question arises if this self-reported data reflects the actually perceived parental care during childhood or if the present context influences this perception. However, promising findings in assessing retest reliability of the PBI suggest that the parental evaluation is a considerably stable measure, not affected by confounding variables like dysthymia, neuroticism, depressive episode, or sex (Plantes et al., 1988; Parker, 1990; Lizardi and Klein, 2005; Wilhelm et al., 2005). Furthermore, good validity of the PBI can be 
concluded from high agreement between sibling ratings (Parker, 1990) as well as from high correlations with according scales in the Adult Attachment Interview (Manassis et al., 1999).

Further, although we have shown that total gray matter volume is not affected by this set of prenatal and postnatal factors, we cannot exclude the possibility that other regions beside the hippocampus are similarly affected. However, the hippocampus has received considerable attention in the past as a brain region significantly affected by stress-related disorders such as posttraumatic stress disorder and depression. Our results contribute to this literature by showing how prenatal and postnatal factors impact on the development of this structure, affecting the individual vulnerability for stress-related disorders.

In summary, the current findings suggest that hippocampal development is influenced by the quality of both prenatal and postnatal environments. We have shown how high quality of maternal care had the potential to counter the effects of prenatal risk on the volume of the hippocampus. At the same time, the results suggest that a disadvantageous environment during prenatal and postnatal periods of brain maturation may contribute to smaller hippocampal volumes in adulthood that have been suggested to increase vulnerability for stress-related disorders in later life (Gilbertson et al., 2002). This is in line with the observation of low birth weight, low maternal care scores on the PBI, and reduced hippocampal volume all being associated with impaired cognitive function and increased risk for psychopathology.

\section{References}

Abernethy LJ, Palaniappan M, Cooke RW (2002) Quantitative magnetic resonance imaging of the brain in survivors of very low birth weight. Arch Dis Child 87:279-283.

Barker DJ, Forsen T, Eriksson JG, Osmond C (2002) Growth and living conditions in childhood and hypertension in adult life: a longitudinal study. J Hypertens 20:1951-1956.

Brabham T, Phelka A, Zimmer C, Nash A, Lopez JF, Vazquez DM (2000) Effects of prenatal dexamethasone on spatial learning and response to stress is influenced by maternal factors. Am J Physiol Regul Integr Comp Physiol 279:R1899-R1909.

Collins DL, Neelin P, Peters TM, Evans AC (1994) Automatic 3D intersubject registration of MR volumetric data in standardized Talairach space. J Comput Assist Tomogr 18:192-205.

Geuze E, Vermetten E, Bremner JD (2005) MR-based in vivo hippocampal volumetrics: 2. Findings in neuropsychiatric disorders. Mol Psychiatry 10:160-184.

Gilbertson MW, Shenton ME, Ciszewski A, Kasai K, Lasko NB, Orr SP, Pitman RK (2002) Smaller hippocampal volume predicts pathologic vulnerability to psychological trauma. Nat Neurosci 5:1242-1247.

Kajantie E, Phillips DI, Andersson S, Barker DJ, Dunkel L, Forsen T, Osmond C, Tuominen J, Wood PJ, Eriksson J (2002) Size at birth, gestational age and cortisol secretion in adult life: foetal programming of both hyper- and hypocortisolism? Clin Endocrinol (Oxf) 57:635-641.

Kramer MS, Platt RW, Wen SW, Joseph KS, Allen A, Abrahamowicz M, Blondel B, Breart G (2001) A new and improved population-based Canadian reference for birth weight for gestational age. Pediatrics 108:E35.

Lemaire V, Lamarque S, Moal ML, Piazza PV, Abrous DN (2006) Postnatal stimulation of the pups counteracts prenatal stress-induced deficits in hippocampal neurogenesis. Biol Psychiatry 59:786-792.

Lizardi H, Klein DN (2005) Long-term stability of parental representations in depressed outpatients utilizing the parental bonding instrument. J Nerv Ment Dis 193:183-188.

Manassis K, Owens M, Adam KS, West M, Sheldon-Keller AE (1999) As- sessing attachment: convergent validity of the adult attachment interview and the parental bonding instrument. Aust N Z J Psychiatry 33:559-567.

Montano MM, Wang MH, vom Saal FS (1993) Sex differences in plasma corticosterone in mouse fetuses are mediated by differential placental transport from the mother and eliminated by maternal adrenalectomy or stress. J Reprod Fertil 99:283-290.

Murphy VE, Gibson PG, Giles WB, Zakar T, Smith R, Bisits AM, Kessell CG, Clifton VL (2003) Maternal asthma is associated with reduced female fetal growth. Am J Respir Crit Care Med 168:1317-1323.

Parker G (1983) Parental "affectionless control" as an antecedent to adult depression. A risk factor delineated. Arch Gen Psychiatry 40:956-960.

Parker G (1990) The parental bonding instrument. A decade of research. Soc Psychiatry Psychiatr Epidemiol 25:281-282.

Parker G, Lipscombe P (1979) Parental characteristics of Jews and Greeks in Australia. Aust N Z J Psychiatry 13:225-229.

Parker G, Tupling H, Brown LB (1979) A parental bonding instrument. Br J Med Psychol 52:1-10.

Phillips DI (1998) Birth weight and the future development of diabetes. A review of the evidence. Diabetes Care 21 [Suppl 2]:B150-B155.

Phillips DI (2004) Fetal programming of the neuroendocrine response to stress: links between low birth weight and the metabolic syndrome. Endocr Res 30:819-826.

Plantes MM, Prusoff BA, Brennan J, Parker G (1988) Parental representations of depressed outpatients from a U.S.A. sample. J Affect Disord 15:149-155.

Pruessner JC, Li LM, Serles W, Pruessner M, Collins DL, Kabani N, Lupien S, Evans AC (2000) Volumetry of hippocampus and amygdala with highresolution MRI and three-dimensional analysis software: minimizing the discrepancies between laboratories. Cereb Cortex 10:433-442.

Pruessner JC, Collins DL, Pruessner M, Evans AC (2001) Age and gender predict volume decline in the anterior and posterior hippocampus in early adulthood. J Neurosci 21:194-200.

Pruessner JC, Baldwin MW, Dedovic K, Renwick R, Mahani NK, Lord C, Meaney M, Lupien S (2005) Self-esteem, locus of control, hippocampal volume, and cortisol regulation in young and old adulthood. NeuroImage 28:815-826.

Reynolds RM, Walker BR, Syddall HE, Andrew R, Wood PJ, Whorwood CB, Phillips DI (2001) Altered control of cortisol secretion in adult men with low birth weight and cardiovascular risk factors. J Clin Endocrinol Metab 86:245-250.

Sapolsky RM, Krey LC, McEwen BS (1986) The neuroendocrinology of stress and aging: the glucocorticoid cascade hypothesis. Endocr Rev $7: 284-301$.

Seckl JR (2004) Prenatal glucocorticoids and long-term programming. Eur J Endocrinol 151 Suppl 3:U49-62.

Teicher MH, Andersen SL, Polcari A, Anderson CM, Navalta CP, Kim DM (2003) The neurobiological consequences of early stress and childhood maltreatment. Neurosci Biobehav Rev 27:33-44.

Vythilingam M, Heim C, Newport J, Miller AH, Anderson E, Bronen R, Brummer M, Staib L, Vermetten E, Charney DS, Nemeroff CB, Bremner JD (2002) Childhood trauma associated with smaller hippocampal volume in women with major depression. Am J Psychiatry 159:2072-2080.

Wadhwa PD, Sandman CA, Porto M, Dunkel-Schetter C, Garite TJ (1993) The association between prenatal stress and infant birth weight and gestational age at birth: a prospective investigation. Am J Obstet Gynecol 169:858-865.

Weinstock M (2005) The potential influence of maternal stress hormones on development and mental health of the offspring. Brain Behav Immun 19:296-308.

Wilhelm K, Niven H, Parker G, Hadzi-Pavlovic D (2005) The stability of the parental bonding instrument over a 20-year period. Psychol Med 35:387-393.

Zhu Z, Li X, Chen W, Zhao Y, Li H, Qing C, Jia N, Bai Z, Liu J (2004) Prenatal stress causes gender-dependent neuronal loss and oxidative stress in rat hippocampus. J Neurosci Res 78:837-844. 